\begin{tabular}{|c|c|}
\hline Title & The Spectrum of the Graded Ring of Differential O perators of a Scored Semigroup A Igebra \\
\hline Author(s) & Saito, Mutsumi \\
\hline Citation & $\begin{array}{l}\text { Communications in A Igebra, 38(3), 829-847 } \\
\text { https://doi.org/10.1080/00927870902828611 }\end{array}$ \\
\hline Issue Date & $2010-03$ \\
\hline Doc URL & http:/hdl.handle.net/2115/44901 \\
\hline Rights & $\begin{array}{l}\text { This is an electronic version of an article published in Communications in A lgebra, 38(3) March 2010, 829-847. } \\
\text { Communications in A lgebra is avail lable online at: } \\
\text { http://www.informaworld.com/smpp/content/db==all ? content=10.1080/00927870902828611 }\end{array}$ \\
\hline Type & article (author version) \\
\hline File Information & CiA 38-3_829-847.pdf \\
\hline
\end{tabular}

Instructions for use 


\title{
THE SPECTRUM OF THE GRADED RING OF DIFFERENTIAL OPERATORS OF A SCORED SEMIGROUP ALGEBRA
}

\author{
MUTSUMI SAITO
}

\begin{abstract}
We describe the set of $\mathbb{Z}^{d}$-graded prime ideals of the graded ring of the ring $D$ of differential operators of a scored semigroup algebra. Moreover we describe the characteristic varieties of $\mathbb{Z}^{d}$-graded critical $D$-modules of a certain type.
\end{abstract}

Mathematics Subject Classification (2000): Primary 13N10; Secondary 16S32, $16 \mathrm{~W} 50$.

Keywords: Prime ideals, scored semigroup algebras, graded ring of differential operators, characteristic varieties

\section{INTRODUCTION}

Let $K$ be an algebraically closed field of characteristic 0 . Let $X$ be an affine algebraic variety over $K$. Since the ring $D(X)$ of differential operators on $X$ is not commutative, to do some algebraic geometry, we need to abelianize $D(X)$. The ring $D(X)$ has the filtration $\left\{D_{k}(X)\right\}$ by the order of differential operators. For a smooth algebraic variety $X$, the abelianization $\operatorname{Gr} D(X)=\bigoplus_{k=0}^{\infty} D_{k}(X) / D_{k-1}(X)$ is the ring of regular functions on the cotangent bundle $T^{*} X$, and the geometric study is crucial for the theory of $D$ modules (for example see (Borel et al, 1987; Hotta, Takeuchi, Tanisaki, 2008; Kashiwara, 2003)). In particular, the characteristic variety is one of the most fundamental invariants for a $D$-module.

Let $A$ be a finite set of column vectors in $\mathbb{Z}^{d}$, and $R_{A}$ the ring of regular funtions on an affine toric variety $X_{A}$ defined by $A$. Then the $\operatorname{ring} \operatorname{Gr} D\left(R_{A}\right)=\operatorname{Gr} D\left(X_{A}\right)$ is Noetherian if and only if $R_{A}$ is scored (Saito, Traves, 2004). The rings $D\left(R_{A}\right)$ and $\operatorname{Gr} D\left(R_{A}\right)$ inherit the natural $\mathbb{Z}^{d}$-grading from $R_{A}$. In this paper, we describe $\mathbb{Z}^{d}$-graded prime ideals of $\operatorname{Gr} D\left(R_{A}\right)$ when $R_{A}$ is scored (Theorem 4.9). Moreover we describe the characteristic varieties of $\mathbb{Z}^{d}$-graded critical $D\left(R_{A}\right)$-modules when they are generated by one $\mathbf{0}$-homogeneous element whose annihilator in $D\left(R_{A}\right)_{\mathbf{0}}=K\left[s_{1}, \ldots, s_{d}\right]$ is a translation by a vector in $K^{d}$ of a prime ideal homogeneous with respect to $s_{1}, \ldots, s_{d}$ (Theorem 6.2).

In Section 2, we recall some fundamental facts about the ring of differential operators of an affine semigroup algebra and the scored property.

Since the semigroup is scored, we are able to reduce the problem to the case $d=1$. In Section 3, we investigate the multiplication structure of a quotient of $\operatorname{Gr} D\left(R_{A}\right)$ in the case $d=1$.

In Section 4, applying the results in Section 3 to the general case, we describe the $\mathbb{Z}^{d}$-graded prime ideals of $\operatorname{Gr} D\left(R_{A}\right)$.

After recalling the $\mathbb{Z}^{d}$-graded critical $D\left(R_{A}\right)$-modules in Section 5 , we describe the characteristic varieties of $\mathbb{Z}^{d}$-graded critical $D\left(R_{A}\right)$-modules of the above type in Section 6.

Date: 30 December 2008. 
The author is grateful to Kohji Yanagawa for fruitful conversations concerning graded ring of differential operators.

\section{The Ring of Differential Operators of an Affine Semigroup Algebra}

In this section, we recall some fundamental facts about the ring of differential operators of an affine semigroup algebra.

Let $A:=\left\{\boldsymbol{a}_{1}, \boldsymbol{a}_{2}, \ldots, \boldsymbol{a}_{n}\right\}$ be a finite set of column vectors in $\mathbb{Z}^{d}$. Sometimes we identify $A$ with the matrix $\left(\boldsymbol{a}_{1}, \boldsymbol{a}_{2}, \ldots, \boldsymbol{a}_{n}\right)$. Let $\mathbb{N} A$ and $\mathbb{R}_{\geq 0} A$ denote the monoid and the cone generated by $A$, respectively. Throughout this paper, we assume that $\mathbb{R}_{\geq_{0}} A$ is strongly convex (for strong convexity, see e.g. (Fulton, 1993, p. 4)), and that $\sum_{j=1}^{n} \mathbb{Z} \boldsymbol{a}_{j}=\mathbb{Z}^{d}$, for simplicity.

Let $K\left[t, t^{-1}\right]$ denote the Laurent polynomial ring $K\left[t_{1}^{ \pm 1}, \ldots, t_{d}^{ \pm 1}\right]$. Then its ring of differential operators $D\left(K\left[t, t^{-1}\right]\right)$ is the ring

$$
K\left[t_{1}^{ \pm 1}, \ldots, t_{d}^{ \pm 1}\right]\left\langle\partial_{1}, \ldots, \partial_{d}\right\rangle,
$$

where $\left[\partial_{i}, t_{j}\right]=\delta_{i j},\left[\partial_{i}, t_{j}^{-1}\right]=-\delta_{i j} t_{j}^{-2}$, and the other pairs of generators commute. Here [, ] denotes the commutator, and $\delta_{i j}$ is 1 if $i=j$ and 0 otherwise. The semigroup algebra $R_{A}:=K[\mathbb{N} A]$ is the subalgebra $\bigoplus_{\boldsymbol{a} \in \mathbb{N} A} K t^{\boldsymbol{a}}$ of $K\left[t, t^{-1}\right]$, where $t^{\boldsymbol{a}}=t_{1}^{a_{1}} t_{2}^{a_{2}} \cdots t_{d}^{a_{d}}$ for $\boldsymbol{a}={ }^{t}\left(a_{1}, a_{2}, \ldots, a_{d}\right)$. The ring of differential operators $D\left(R_{A}\right)$ is a subring of $D\left(K\left[t, t^{-1}\right]\right)$, namely

$$
D\left(R_{A}\right)=\left\{P \in K\left[t_{1}^{ \pm 1}, \ldots, t_{d}^{ \pm 1}\right]\left\langle\partial_{1}, \ldots, \partial_{d}\right\rangle: P\left(R_{A}\right) \subseteq R_{A}\right\} .
$$

Put $s_{j}:=t_{j} \partial_{j}$ for $j=1,2, \ldots, d$. Then it is easy to see that $s_{j} \in D\left(R_{A}\right)$ for all $j$. We introduce a $\mathbb{Z}^{d}$-grading on the ring $D\left(R_{A}\right)$; for $\boldsymbol{a}={ }^{t}\left(a_{1}, a_{2}, \ldots, a_{d}\right) \in \mathbb{Z}^{d}$, set

$$
D\left(R_{A}\right)_{\boldsymbol{a}}:=\left\{P \in D\left(R_{A}\right):\left[s_{j}, P\right]=a_{j} P \quad \text { for } j=1,2, \ldots, d\right\} .
$$

Then $D\left(R_{A}\right)=\bigoplus_{\boldsymbol{a} \in \mathbb{Z}^{d}} D\left(R_{A}\right)_{\boldsymbol{a}}$. Given $\boldsymbol{a} \in \mathbb{Z}^{d}$, it is not difficult to see that there exists an ideal $I$ of $K[s]:=K\left[s_{1}, \ldots, s_{d}\right]$ such that $D\left(R_{A}\right)_{\boldsymbol{a}}=t^{\boldsymbol{a}} I$. To describe this ideal $I$ explicitly, we define a subset $\Omega(\boldsymbol{a})$ of the semigroup $\mathbb{N} A$ by

$$
\Omega(\boldsymbol{a})=\{\boldsymbol{b} \in \mathbb{N} A: \boldsymbol{b}+\boldsymbol{a} \notin \mathbb{N} A\}=\mathbb{N} A \backslash(-\boldsymbol{a}+\mathbb{N} A) .
$$

Then $D\left(R_{A}\right)_{\boldsymbol{a}}$ is described as follows.

Theorem 2.1 (Musson, 1987, Theorem 2.3).

$$
D\left(R_{A}\right)_{\boldsymbol{a}}=t^{\boldsymbol{a}} \mathbb{I}(\Omega(\boldsymbol{a})) \quad \text { for all } \boldsymbol{a} \in \mathbb{Z}^{d},
$$

where

$$
\mathbb{I}(\Omega(\boldsymbol{a})):=\{f(s) \in K[s]: f \text { vanishes on } \Omega(\boldsymbol{a})\}
$$

In particular, $D\left(R_{A}\right)_{\boldsymbol{a}}=t^{\boldsymbol{a}} K[s]=K[s] t^{\boldsymbol{a}}$ for each $\boldsymbol{a} \in \mathbb{N} A$, since $\Omega(\boldsymbol{a})=\emptyset$ in this case.

Next we explain the order filtration. A differential operator

$$
P=\sum_{\boldsymbol{a} \in \mathbb{N}^{d}} a_{\boldsymbol{a}}(t) \partial^{\boldsymbol{a}} \in D\left(K\left[t, t^{-1}\right]\right)
$$

is said to be of order $k$ if $a_{\boldsymbol{a}} \neq 0$ for some $\boldsymbol{a}$ with $|\boldsymbol{a}|=k$ and $a_{\boldsymbol{a}}=0$ for all $\boldsymbol{a}$ with $|\boldsymbol{a}|>k$, where $|\boldsymbol{a}|=a_{1}+a_{2}+\cdots+a_{d}$. Let $D_{k}\left(R_{A}\right)$ denote the set of differential operators in 
$D\left(R_{A}\right)$ of order at most $k$. Then $\left\{D_{k}\left(R_{A}\right)\right\}_{k \in \mathbb{N}}$ is called the order filtration of $D\left(R_{A}\right)$. We consider the graded ring $G:=\operatorname{Gr}\left(D\left(R_{A}\right)\right)$ of $D\left(R_{A}\right)$ with respect to the order filtration:

$$
G:=\operatorname{Gr}\left(D\left(R_{A}\right)\right):=\bigoplus_{k \in \mathbb{N}} D_{k}\left(R_{A}\right) / D_{k-1}\left(R_{A}\right)
$$

where we put $D_{-1}\left(R_{A}\right)=0$. For an operator $P$ of order $k, \bar{P}$ denotes the element of $D_{k}\left(R_{A}\right) / D_{k-1}\left(R_{A}\right)$ represented by $P$. Sometimes we write simply $P$ instead of $\bar{P}$, especially when $P=t^{a}$, or $P=s_{i}$. The graded $\operatorname{ring} G$ is a subring of the commutative ring

$$
\operatorname{Gr}\left(D\left(K\left[t, t^{-1}\right]\right)\right)=K\left[t_{1}^{ \pm 1}, t_{2}^{ \pm 1}, \ldots, t_{d}^{ \pm 1}, \xi_{1}, \xi_{2}, \ldots, \xi_{d}\right]
$$

where $\xi_{j}=\overline{\partial_{j}}$. Since each $D_{k}\left(R_{A}\right)$ is $\mathbb{Z}^{d}$-graded by $D_{k}\left(R_{A}\right)=\bigoplus_{\boldsymbol{d} \in \mathbb{Z}^{d}} D_{k}\left(R_{A}\right) \cap D\left(R_{A}\right)_{\boldsymbol{d}}$, the graded ring $G$ inherits the $\mathbb{Z}^{d}$-grading:

$$
G=\bigoplus_{d \in \mathbb{Z}^{d}} G_{\boldsymbol{d}}
$$

In (Saito, Traves, 2004), we proved that $G=\operatorname{Gr}(D(K[\mathbb{N} A])$ ) is Noetherian if and only if the semigroup $\mathbb{N} A$ is scored.

Next we recall the definition of a scored semigroup from (Saito, Traves, 2001). We denote by $\mathcal{F}$ the set of facets (maximal proper faces) of the cone $\mathbb{R}_{\geq 0} A$. Given $\sigma \in \mathcal{F}$, we denote by $F_{\sigma}$ the primitive integral support function of $\sigma$, i.e., $F_{\sigma}$ is the linear form on $\mathbb{R}^{d}$ uniquely determined by the conditions:

(1) $F_{\sigma}\left(\mathbb{R}_{\geq 0} A\right) \geq 0$

(2) $F_{\sigma}(\sigma)=0$

(3) $F_{\sigma}\left(\mathbb{Z}^{d}\right)=\mathbb{Z}$

Definition 2.2. The semigroup $\mathbb{N} A$ is said to be scored if

$$
\mathbb{N} A=\bigcap_{\sigma \in \mathcal{F}}\left\{\boldsymbol{a} \in \mathbb{Z}^{d}: F_{\sigma}(\boldsymbol{a}) \in F_{\sigma}(\mathbb{N} A)\right\} .
$$

Example 2.3. Let

Then

$$
A_{1}=\left(\boldsymbol{a}_{1}, \boldsymbol{a}_{2}, \boldsymbol{a}_{3}\right)=\left(\begin{array}{ccc}
1 & 1 & 1 \\
0 & 2 & 3
\end{array}\right)
$$

$$
\begin{gathered}
\mathcal{F}=\left\{\sigma_{1}=\mathbb{R}_{\geq 0} \boldsymbol{a}_{1}, \sigma_{3}=\mathbb{R}_{\geq 0} \boldsymbol{a}_{3}\right\}, \\
F_{\sigma_{1}}\left(s_{1}, s_{2}\right)=s_{2}, F_{\sigma_{3}}\left(s_{1}, s_{2}\right)=3 s_{1}-s_{2}, \text { and } \\
\mathbb{N} \backslash F_{\sigma_{1}}\left(\mathbb{N} A_{1}\right)=\{1\}, \quad \mathbb{N} \backslash F_{\sigma_{3}}\left(\mathbb{N} A_{1}\right)=\emptyset .
\end{gathered}
$$

As illustrated in Figure 1, the semigroup $\mathbb{N} A_{1}$ is scored.

Next let

$$
A_{2}=\left(\boldsymbol{a}_{1}, \boldsymbol{a}_{2}, \boldsymbol{a}_{3}\right)=\left(\begin{array}{ccc}
2 & 0 & 1 \\
0 & 1 & 1
\end{array}\right) .
$$

Then

$$
\begin{aligned}
& \mathcal{F}=\left\{\sigma_{1}=\mathbb{R}_{\geq 0} \boldsymbol{a}_{1}, \sigma_{2}=\mathbb{R}_{\geq 0} \boldsymbol{a}_{2}\right\}, \\
& F_{\sigma_{1}}\left(s_{1}, s_{2}\right)=s_{2}, F_{\sigma_{2}}\left(s_{1}, s_{2}\right)=s_{1}, \text { and } \\
& F_{\sigma_{1}}\left(\mathbb{N} A_{2}\right)=\mathbb{N}, \quad F_{\sigma_{2}}\left(\mathbb{N} A_{2}\right)=\mathbb{N} .
\end{aligned}
$$

Since $\mathbb{N} A_{2} \neq \mathbb{N}^{2}$, the semigroup $\mathbb{N} A_{2}$ is not scored. 


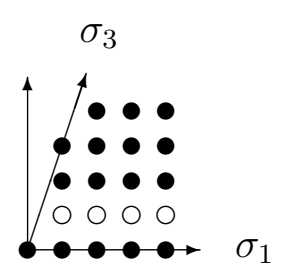

$\mathbb{N} A_{1}$

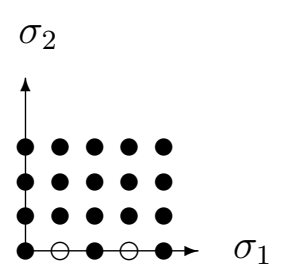

$\mathbb{N} A_{2}$

FiguRE 1. The semigroups in Example 2.3

Corollary 2.4 (Saito, Traves, 2004, Proposition 6.1). Suppose that the semigroup $\mathbb{N} A$ is scored. Then

$$
D\left(R_{A}\right)_{\boldsymbol{a}}=P_{\boldsymbol{a}} K[s]
$$

where

$$
P_{\boldsymbol{a}}:=t^{\boldsymbol{a}} p_{a}(s), \quad p_{\boldsymbol{a}}(s):=\prod_{\sigma \in \mathcal{F}} \prod_{m \in F_{\sigma}(\mathbb{N} A) \backslash\left(-F_{\sigma}(\boldsymbol{a})+F_{\sigma}(\mathbb{N} A)\right)}\left(F_{\sigma}(s)-m\right) .
$$

Proof. We have

$$
\Omega(\boldsymbol{a})=\mathbb{N} A \backslash(-\boldsymbol{a}+\mathbb{N} A)=\bigcup_{\sigma \in \mathcal{F}}\left\{\boldsymbol{b} \in \mathbb{N} A: F_{\sigma}(\boldsymbol{b}) \notin-F_{\sigma}(\boldsymbol{a})+F_{\sigma}(\mathbb{N} A)\right\},
$$

since $-\boldsymbol{a}+\mathbb{N} A=\bigcap_{\sigma \in \mathcal{F}}\left\{\boldsymbol{b} \in \mathbb{Z}^{d}: F_{\sigma}(\boldsymbol{b}) \in-F_{\sigma}(\boldsymbol{a})+F_{\sigma}(\mathbb{N} A)\right\}$ by (2.6). Hence the ideal $\mathbb{I}(\Omega(\boldsymbol{a}))$ of $K[s]=K\left[s_{1}, \ldots, s_{d}\right]$ is generated by the single polynomial $p_{\boldsymbol{a}}(s)$, and the assertion follows from Theorem 2.1.

The following corollary is the graded version of Corollary 2.4 with respect to the order filtration (see (2.4)):

Corollary 2.5 (Saito, Traves, 2004, Corollary 6.2). Suppose that the semigroup $\mathbb{N} A$ is scored. Then the $\mathbb{Z}^{d}$-grading (2.5) of $G=\operatorname{Gr}\left(D\left(R_{A}\right)\right)$ is described as follows:

$$
\begin{aligned}
G & =\bigoplus_{\boldsymbol{a} \in \mathbb{Z}^{d}} \overline{t^{\boldsymbol{a}} \mathbb{I}(\Omega(\boldsymbol{a}))}=\bigoplus_{\boldsymbol{a} \in \mathbb{Z}^{d}} \bar{P}_{\boldsymbol{a}} K[s] \\
& \subseteq K\left[t_{1}^{ \pm}, \ldots, t_{d}^{ \pm}, \xi_{1}, \ldots, \xi_{d}\right]=\operatorname{Gr}\left(D\left(K\left[t, t^{-1}\right]\right)\right),
\end{aligned}
$$

and

$$
\bar{P}_{\boldsymbol{a}}=t^{\boldsymbol{a}} \cdot \prod_{\sigma \in \mathcal{F}} F_{\sigma}(s)^{\sharp\left(F_{\sigma}(\mathbb{N} A) \backslash\left(-F_{\sigma}(\boldsymbol{a})+F_{\sigma}(\mathbb{N} A)\right)\right)} .
$$

From now on, we always assume that the semigroup $\mathbb{N} A$ is scored.

Let $\operatorname{Spec}_{\mathbb{Z}^{d}}(G)$ denote the set of $\mathbb{Z}^{d}$-graded prime ideals of $G$. Since $G_{\mathbf{0}}(=K[s])$ is a subalgebra of $G$, we have a natural map

$$
\pi: \operatorname{Spec}_{\mathbb{Z}^{d}}(G) \ni \mathfrak{P}=\bigoplus_{\boldsymbol{a} \in \mathbb{Z}^{d}} \mathfrak{P}_{\boldsymbol{a}} \mapsto \mathfrak{P}_{\mathbf{0}} \in \operatorname{Spec}(K[s]) .
$$

Given a prime ideal $\mathfrak{p} \in \operatorname{Spec}(K[s])$, we shall describe its fiber $\pi^{-1}(\mathfrak{p})$. 


\section{THE CASE $d=1$}

In this section, we assume $d=1$. In this case, $\mathbb{R}_{\geq 0} A=\mathbb{R}_{\geq 0}$ or $-\mathbb{R}_{\geq 0}$ by the strong convexity. We assume that $\mathbb{R}_{\geq 0} A=\mathbb{R}_{\geq 0}$. The cone $\mathbb{R}_{\geq 0}$ has only one facet $\{0\}, F_{\{0\}}(s)=s$, and $\mathbb{N} A$ is always scored. We consider the multiplication structure of $G / G s=\bigoplus_{a \in \mathbb{Z}} K \overline{P_{a}}$, where $P_{a}$ was defined in Corollary 2.4. Throughout this section, we set

$$
S:=\mathbb{N} A \text {. }
$$

Since we assume that $\mathbb{Z} A=\mathbb{Z}$, the set $\mathbb{N} \backslash S$ is finite. We also set

$$
\mathbb{N} \backslash S=\left\{c_{1}<\cdots<c_{h}\right\}
$$

throughout this section. Then for $a \in \mathbb{Z}$

$$
\begin{aligned}
\Omega(a)= & S \backslash(-a+S) \\
= & \left(\{n \in \mathbb{N}: n<-a\} \cup\left\{-a+c_{r} \in \mathbb{N}: 1 \leq r \leq h\right\}\right) \\
& \\
& \backslash\left\{c_{1}, \ldots, c_{h}\right\} .
\end{aligned}
$$

The following lemma is clear from (3.3).

Lemma 3.1. Let $a \in \mathbb{N}$. Then

(1) $\Omega(a)=\left\{c_{r}-a \in S: 1 \leq r \leq h\right\}$,

(2) $\Omega(-a)=\{n \in S: n<a\} \cup\left\{a+c_{r} \in S: 1 \leq r \leq h\right\}$.

Notation 3.2. For $a \in \mathbb{Z}$, set

$$
\operatorname{deg}(a):=\sharp \Omega(a) .
$$

By definition (see Corollary 2.4),

$$
p_{a}=\prod_{k \in \Omega(a)}(s-k)
$$

and hence $\operatorname{deg}(a)=\operatorname{deg}\left(p_{a}\right)$.

Corollary 3.3. Let $a \in \mathbb{N}$. Then $a \in S$ if and only if $\operatorname{deg}(a)=0$.

Proof. Since $S$ is a semigroup, $a \in S$ implies $\Omega(a)=\emptyset$. If $a \notin S$, that is $a=c_{i}$ for some $i$, then $0 \in \Omega(a)$ by Lemma $3.1(1)$.

Proposition 3.4. Let $a \in \mathbb{N}$. Then

$$
\operatorname{deg}(-a)=a+\operatorname{deg}(a)
$$

Proof. Let $c_{i}<a \leq c_{i+1}$. (Here $c_{0}=-1, c_{h+1}=+\infty$.) We have $\sharp\{n \in S: n<a\}=a-i$, and

$$
\begin{aligned}
\sharp\left\{r: a+c_{r} \in S\right\} & =h-\sharp\left\{r: a+c_{r} \notin S\right\} \\
& \left.=h-\sharp\left\{l \geq i+1: c_{l}=a+c_{r} \quad \text { (for some } r\right)\right\} \\
& =h-\sharp\left\{l \geq i+1: c_{l}-a \notin S\right\} \\
& =h-\left((h-i)-\sharp\left\{l \geq i+1: c_{l}-a \in S\right\}\right) \\
& =i+\sharp\left\{l \geq i+1: c_{l}-a \in S\right\} \\
& =i+\operatorname{deg}(a) .
\end{aligned}
$$

Hence, by Lemma $3.1(2), \operatorname{deg}(-a)=a+\operatorname{deg}(a)$. 
For $a \in \mathbb{Z}$, we call

$$
\operatorname{expdeg}(a)= \begin{cases}0 & \text { if } a \geq 0 \\ |a| & \text { if } a \leq 0\end{cases}
$$

the expected degree of $a$.

Corollary 3.5. Let $a \in \mathbb{Z}$. Then we have the following:

(1) $\operatorname{deg}(a) \geq \operatorname{expdeg}(a)$.

(2) $\operatorname{deg}(a)=\operatorname{expdeg}(a)$ if and only if $|a| \in S$.

Proof. Let $a \in \mathbb{N}$. Then by definition $\operatorname{deg}(a)=\sharp \Omega(a) \geq 0=\operatorname{expdeg}(a)$, and $\operatorname{deg}(a)=0$ if and only if $a \in S$ by Corollary 3.3.

By Proposition 3.4, $\operatorname{deg}(-a)=a+\operatorname{deg}(a) \geq a=\operatorname{expdeg}(-a)$, and the equality holds if and only if $a \in S$ again by Corollary 3.3.

Theorem 3.6. Let $a, b \in \mathbb{Z}$. Then

(1) $\operatorname{deg}(a)+\operatorname{deg}(b) \geq \operatorname{deg}(a+b)$.

(2) For $a, b \neq 0, \operatorname{deg}(a)+\operatorname{deg}(b)=\operatorname{deg}(a+b)$ if and only if $a, b \in S$ or $a, b \in-S$.

(3) If $a=0$ or $b=0$, then $\operatorname{deg}(a)+\operatorname{deg}(b)=\operatorname{deg}(a+b)$.

Proof. Since $D\left(R_{A}\right)$ is a subalgebra of an integral domain $D\left(K\left[t, t^{-1}\right]\right)(2.1), D\left(R_{A}\right)$ is also an integral domain. Recall that $D\left(R_{A}\right)_{a}=t^{a} p_{a} K[s]$ (Corollary 2.4). We have $0 \neq$ $t^{a} p_{a} \cdot t^{b} p_{b} \in D\left(R_{A}\right)_{a+b}=t^{a+b} p_{a+b} K[s]$. Recalling that $\operatorname{deg}(c)=\operatorname{deg}\left(p_{c}\right)$ for all $c \in \mathbb{Z}$ (see (3.4)), we have $\operatorname{deg}(a)+\operatorname{deg}(b) \geq \operatorname{deg}(a+b)$.

(3) follows from $\operatorname{deg}(0)=0$.

By Corollary 3.3 and Proposition 3.4, the if-part of (2) holds.

We prove the only-if part of (2) in Lemmas 3.7, 3.9, and 3.11.

Lemma 3.7. Let $a, b \in \mathbb{Z}_{>0}$. If $a \notin S$, then $\operatorname{deg}(a)+\operatorname{deg}(b)>\operatorname{deg}(a+b)$.

Proof. We divide the proof into three cases.

(Case 1) Suppose $a+b \in S$. Then by Corollary $3.3 \operatorname{deg}(a+b)=0$ and $\operatorname{deg}(a)>0$.

(Case 2) Suppose $a+b \notin S$ and $b \in S$. By Corollary 3.3 $\operatorname{deg}(b)=0$. Let $a=c_{i}$ and $a+b=c_{j}$ with $i<j$.

Suppose $c_{r}-c_{j} \in S$. Note that, in this case, $c_{r}-c_{j} \in \Omega(a+b)$. Then $c_{r}-c_{i}=$ $\left(c_{r}-c_{j}\right)+b \in S$. Hence $\sharp \Omega\left(c_{j}\right) \leq \sharp \Omega\left(c_{i}\right)$ by Lemma 3.1 (1). Furthermore, since $c_{i}-c_{j} \notin S$, we have $\sharp \Omega\left(c_{j}\right)<\sharp \Omega\left(c_{i}\right)$. We thus have $\operatorname{deg}(a+b)=\operatorname{deg}\left(c_{j}\right)<\operatorname{deg}\left(c_{i}\right)=\operatorname{deg}(a)+0=$ $\operatorname{deg}(a)+\operatorname{deg}(b)$.

(Case 3) Suppose $a+b \notin S$ and $b \notin S$. Let $a=c_{i}, b=c_{i^{\prime}}$, and $a+b=c_{j}$ with $i, i^{\prime}<j$.

We show the following:

\section{Claim 3.8.}

$$
\begin{aligned}
& \left\{c_{r}: c_{r}-c_{j} \in S, r \neq j\right\} \\
& \subseteq\left\{c_{r}: c_{r}-c_{i^{\prime}} \in S, r \neq i^{\prime}\right\} \coprod\left\{c_{l}+c_{i^{\prime}}: c_{l}-c_{i} \in S, r \neq i\right\} .
\end{aligned}
$$

(Proof of Claim 3.8) Suppose $c_{r}-c_{i^{\prime}} \in S$ and $c_{r}=c_{l}+c_{i^{\prime}}$. Then $c_{l} \in S$, which is a contradiction. Hence the union is disjoint.

Next suppose $c_{r}-c_{j} \in S(r>j)$ and $c_{r}-c_{i^{\prime}} \notin S$. Let $c_{l}=c_{r}-c_{i^{\prime}}$. Then $c_{r}=c_{l}+c_{i^{\prime}}$, and $c_{j}=c_{i}+c_{i^{\prime}}$. Since $S \ni c_{r}-c_{j}=\left(c_{r}-c_{i^{\prime}}\right)-c_{i}=c_{l}-c_{i}$, the claim has been proved. 
(Continuation of the proof of Lemma 3.7) By Lemma 3.1 (1) and Claim 3.8, $\operatorname{deg}\left(c_{j}\right)-$ $1 \leq\left(\operatorname{deg}\left(c_{i}\right)-1\right)+\left(\operatorname{deg}\left(c_{i^{\prime}}\right)-1\right)$, hence $\operatorname{deg}\left(c_{j}\right)<\operatorname{deg}\left(c_{i}\right)+\operatorname{deg}\left(c_{i^{\prime}}\right)$.

Lemma 3.9. If $a, b \in \mathbb{Z}, a>b>0$, then $\operatorname{deg}(a)+\operatorname{deg}(-b)>\operatorname{deg}(a-b)$.

Proof. First we show the following:

Claim 3.10.

$$
\begin{aligned}
& \left\{c_{r}-(a-b) \in S\right\} \\
& \subseteq\{n \in S: n<b\} \coprod\left\{c_{r}+b \in S\right\} \coprod\left\{c_{r}-a+b: c_{r}-a \in S\right\} .
\end{aligned}
$$

Here by Lemma 3.1 the number of the elements of the set on the left hand side is $\operatorname{deg}(a-b)$, while the union of the first two sets on the right hand side has $\operatorname{deg}(-b)$ elements, and the third set has $\operatorname{deg}(a)$ elements.

(Proof of Claim 3.10) It is clear that the union is disjoint.

Let $c_{r}-(a-b) \in S$. If this does not belong to the first set on the right hand side, then $c_{r} \geq a$. If $c_{r}-(a-b) \in S$ does not belong to the second set either, then $c_{r}-(a-b)-b \in S$, or $c_{r}-a \in S$. Hence $c_{r}-(a-b)=c_{r}-a+b$ belongs to the third set.

(Continuation of the proof of Lemma 3.9) $c_{h}+b$ does not belong to the left hand side of Claim 3.10, but belongs to (the second set of) the right hand side. Hence $\operatorname{deg}(a-b)<$ $\operatorname{deg}(-b)+\operatorname{deg}(a)$.

Lemma 3.11. $\operatorname{deg}(a)+\operatorname{deg}(-b)>\operatorname{deg}(a-b)$ for $b>a>0$.

Proof. By Proposition 3.4 and Lemma 3.9, $\operatorname{deg}(a)+\operatorname{deg}(-b)=\operatorname{deg}(-a)-a+\operatorname{deg}(b)+b>$ $\operatorname{deg}(b-a)+b-a=\operatorname{deg}(a-b)$.

\section{General Case}

We return to the general case. In this section, applying the results in Section 3 to the general case, for $\mathfrak{p} \in \operatorname{Spec}(K[s])$, we describe its fiber $\pi^{-1}(\mathfrak{p})$ of $\pi(2.8)$, the set of $\mathbb{Z}^{d}$-graded prime ideals $\mathfrak{P}$ of $G$ with $\mathfrak{P}_{0}=\mathfrak{p}$.

For a facet $\sigma \in \mathcal{F}$, let

$$
\mathbb{N} \backslash F_{\sigma}(\mathbb{N} A)=\left\{c(\sigma)_{1}<\cdots<c(\sigma)_{h(\sigma)}\right\} .
$$

We can apply the results in $\S 3$ to the subsemigroup $F_{\sigma}(\mathbb{N} A)$ of $\mathbb{N}$. For $\sigma \in \mathcal{F}$ and $\boldsymbol{a} \in \mathbb{Z}^{d}$, as in Notation 3.2 and (3.5), set

$$
\begin{aligned}
\operatorname{deg}_{\sigma}(\boldsymbol{a}) & :=\sharp\left(F_{\sigma}(\mathbb{N} A) \backslash\left(-F_{\sigma}(\boldsymbol{a})+F_{\sigma}(\mathbb{N} A)\right)\right), \\
\operatorname{expdeg}_{\sigma}(\boldsymbol{a}) & := \begin{cases}0 & \text { if } F_{\sigma}(\boldsymbol{a}) \geq 0 \\
\left|F_{\sigma}(\boldsymbol{a})\right| & \text { if } F_{\sigma}(\boldsymbol{a}) \leq 0 .\end{cases}
\end{aligned}
$$

By Corollary 2.5, we have

$$
\overline{P_{a}}=t^{a} \prod_{\sigma \in \mathcal{F}} F_{\sigma}^{\operatorname{deg}_{\sigma}(a)}
$$

Lemma 3.1, Corollary 3.3, Proposition 3.4, Corollary 3.5, and Theorem 3.6 read as follows: 
Lemma 4.1. Let $\boldsymbol{a} \in \mathbb{Z}^{d}$.

$$
\begin{aligned}
& F_{\sigma}(\mathbb{N} A) \backslash\left(-F_{\sigma}(\boldsymbol{a})+F_{\sigma}(\mathbb{N} A)\right) \\
& =\left\{\begin{array}{r}
\left\{c(\sigma)_{r}-F_{\sigma}(\boldsymbol{a}) \in F_{\sigma}(\mathbb{N} A): 1 \leq r \leq h(\sigma)\right\} \quad \text { if } F_{\sigma}(\boldsymbol{a}) \geq 0, \\
\left\{c(\sigma)_{r}-F_{\sigma}(\boldsymbol{a}) \in F_{\sigma}(\mathbb{N} A): 1 \leq r \leq h(\sigma)\right\} \\
\cup\left\{n \in F_{\sigma}(\mathbb{N} A): n<-F_{\sigma}(\boldsymbol{a})\right\} \quad \text { if } F_{\sigma}(\boldsymbol{a})<0 .
\end{array}\right.
\end{aligned}
$$

Corollary 4.2. Let $\boldsymbol{a} \in \mathbb{Z}^{d}$. Then $F_{\sigma}(\boldsymbol{a}) \in F_{\sigma}(\mathbb{N} A)$ if and only if $\operatorname{deg}_{\sigma}(\boldsymbol{a})=0$.

Proposition 4.3. Let $\boldsymbol{a} \in \mathbb{Z}^{d}$. If $F_{\sigma}(\boldsymbol{a}) \geq 0$, then

$$
\operatorname{deg}_{\sigma}(-\boldsymbol{a})=F_{\sigma}(\boldsymbol{a})+\operatorname{deg}_{\sigma}(\boldsymbol{a}) .
$$

Corollary 4.4. Let $\boldsymbol{a} \in \mathbb{Z}^{d}$. Then we have the following:

(1) $\operatorname{deg}_{\sigma}(\boldsymbol{a}) \geq \operatorname{expdeg}_{\sigma}(\boldsymbol{a})$.

(2) $\operatorname{deg}_{\sigma}(\boldsymbol{a})=\operatorname{expdeg}_{\sigma}(\boldsymbol{a})$ if and only if $\left|F_{\sigma}(\boldsymbol{a})\right| \in F_{\sigma}(\mathbb{N} A)$.

Theorem 4.5. Let $\boldsymbol{a}, \boldsymbol{b} \in \mathbb{Z}^{d}$.

(1) $\operatorname{deg}_{\sigma}(\boldsymbol{a})+\operatorname{deg}_{\sigma}(\boldsymbol{b}) \geq \operatorname{deg}_{\sigma}(\boldsymbol{a}+\boldsymbol{b})$.

(2) Suppose that $F_{\sigma}(\boldsymbol{a}), F_{\sigma}(\boldsymbol{b}) \neq 0$. Then $\operatorname{deg}_{\sigma}(\boldsymbol{a})+\operatorname{deg}_{\sigma}(\boldsymbol{b})=\operatorname{deg}_{\sigma}(\boldsymbol{a}+\boldsymbol{b})$ if and only if $F_{\sigma}(\boldsymbol{a}), F_{\sigma}(\boldsymbol{b}) \in F_{\sigma}(\mathbb{N} A)$ or $F_{\sigma}(\boldsymbol{a}), F_{\sigma}(\boldsymbol{b}) \in-F_{\sigma}(\mathbb{N} A)$.

(3) If $F_{\sigma}(\boldsymbol{a})=0$ or $F_{\sigma}(\boldsymbol{b})=0$, then $\operatorname{deg}_{\sigma}(\boldsymbol{a})+\operatorname{deg}_{\sigma}(\boldsymbol{b})=\operatorname{deg}_{\sigma}(\boldsymbol{a}+\boldsymbol{b})$.

Let $\mathfrak{p}$ be a prime ideal of $K[s]=K\left[s_{1}, \ldots, s_{d}\right]$. Define a set $\mathcal{F}(\mathfrak{p})$ of facets by

$$
\mathcal{F}(\mathfrak{p})=\left\{\sigma \in \mathcal{F}: F_{\sigma} \in \mathfrak{p}\right\} .
$$

Let $\Sigma(\mathfrak{p})$ denote the fan determined by the hyperplane arrangement

$$
\{\mathbb{R} \sigma: \sigma \in \mathcal{F}(\mathfrak{p})\},
$$

i.e., $\Sigma(\mathfrak{p})$ is the set of intersections

$$
\bigcap_{\sigma \in \mathcal{F}^{\prime}}\left(F_{\sigma} \geq 0\right) \cap \bigcap_{\sigma \in \mathcal{F}^{\prime \prime}}\left(F_{\sigma} \leq 0\right)
$$

for subsets $\mathcal{F}^{\prime}, \mathcal{F}^{\prime \prime}$ of $\mathcal{F}(\mathfrak{p})$ with $\mathcal{F}^{\prime} \cup \mathcal{F}^{\prime \prime}=\mathcal{F}(\mathfrak{p})$. For fans, see e.g. (Fulton, 1993).

Set

$$
S(\mathfrak{p}):=\left\{\boldsymbol{a} \in \mathbb{Z}^{d}:\left|F_{\sigma}(\boldsymbol{a})\right| \in F_{\sigma}(\mathbb{N} A) \text { for all } \sigma \in \mathcal{F}(\mathfrak{p})\right\} .
$$

For $\boldsymbol{a} \in \mathbb{Z}^{d}$, we define the degree and the expected degree of $\boldsymbol{a}$ with respect to $\mathfrak{p}$ by

$$
\begin{aligned}
\operatorname{deg}_{\mathfrak{p}}(\boldsymbol{a}) & :=\sum_{\sigma \in \mathcal{F}(\mathfrak{p})} \operatorname{deg}_{\sigma}(\boldsymbol{a}), \\
\operatorname{expdeg}(\boldsymbol{a}) & :=\sum_{\sigma \in \mathcal{F}(\mathfrak{p})} \operatorname{expdeg}_{\sigma}(\boldsymbol{a}) .
\end{aligned}
$$

Then by Corollary 2.4

$$
\operatorname{deg}_{\mathfrak{m}}(\boldsymbol{a})=\operatorname{deg}\left(p_{\boldsymbol{a}}\right),
$$

where $\mathfrak{m}=\left(s_{1}, \ldots, s_{d}\right)$. By Corollary 4.4 , we have the following corollary.

Corollary 4.6. Let $\boldsymbol{a} \in \mathbb{Z}^{d}$. Let $\mathfrak{p}$ be a prime ideal of $K[s]$.

(1) $\operatorname{deg}_{\mathfrak{p}}(\boldsymbol{a}) \geq \operatorname{expdeg}(\boldsymbol{a})$. 
(2) $\operatorname{deg}_{\mathfrak{p}}(\boldsymbol{a})=\operatorname{expdeg}(\boldsymbol{a})$ if and only if $\boldsymbol{a} \in S(\mathfrak{p})$.

Recall from Corollary 2.5 that

$$
G=\operatorname{Gr}\left(D\left(R_{A}\right)\right)=\bigoplus_{\boldsymbol{a} \in \mathbb{Z}^{d}} \bar{P}_{\boldsymbol{a}} K[s] .
$$

Lemma 4.7. Let $\boldsymbol{a}, \boldsymbol{b} \in \mathbb{Z}^{d}$. Let $\mathfrak{p}$ be a prime ideal of $K[s]$. Then

(1) $\overline{P_{\boldsymbol{a}}} \cdot \overline{P_{\boldsymbol{b}}} \in G \mathfrak{p}$ unless $\boldsymbol{a}$ and $\boldsymbol{b}$ belong to the same cone in $\Sigma(\mathfrak{p})$.

(2) $\overline{P_{\boldsymbol{a}}} \cdot \overline{P_{\boldsymbol{b}}} \notin G \mathfrak{p}$ if $\boldsymbol{a}, \boldsymbol{b} \in S(\mathfrak{p}) \cap \tau$ for some cone $\tau \in \Sigma(\mathfrak{p})$.

(3) Let $\boldsymbol{a}, \boldsymbol{b} \in \tau \in \Sigma(\mathfrak{p})$. Then $\overline{P_{\boldsymbol{a}}} \cdot \overline{P_{\boldsymbol{b}}} \notin G \mathfrak{p}$ if and only if $\left|F_{\sigma}(\boldsymbol{a})\right|,\left|F_{\sigma}(\boldsymbol{b})\right| \in F_{\sigma}(\mathbb{N} A)$ for all $\sigma \in \mathcal{F}(\mathfrak{p})$ with $F_{\sigma}(\boldsymbol{a}), F_{\sigma}(\boldsymbol{b}) \neq 0$.

Proof. By (4.4)

$$
\overline{P_{\boldsymbol{a}}} \cdot \overline{P_{\boldsymbol{b}}}=\overline{P_{\boldsymbol{a}+\boldsymbol{b}}} \prod_{\sigma \in \mathcal{F}} F_{\sigma}^{\operatorname{deg}_{\sigma}(\boldsymbol{a})+\operatorname{deg}_{\sigma}(\boldsymbol{b})-\operatorname{deg}_{\sigma}(\boldsymbol{a}+\boldsymbol{b})} .
$$

(Here $\operatorname{deg}_{\sigma}(\boldsymbol{a})+\operatorname{deg}_{\sigma}(\boldsymbol{b})-\operatorname{deg}_{\sigma}(\boldsymbol{a}+\boldsymbol{b}) \geq 0$ by Theorem 4.5 (1).) Hence $\overline{P_{\boldsymbol{a}}} \cdot \overline{P_{\boldsymbol{b}}} \in G \mathfrak{p}$ if and only if there exists a facet $\sigma \in \mathcal{F}(\mathfrak{p})$ such that $\operatorname{deg}_{\sigma}(\boldsymbol{a})+\operatorname{deg}_{\sigma}(\boldsymbol{b})>\operatorname{deg}_{\sigma}(\boldsymbol{a}+\boldsymbol{b})$ (see (4.5) for the definition of $\mathcal{F}(\mathfrak{p}))$.

(1) Suppose that $\boldsymbol{a}$ and $\boldsymbol{b}$ does not belong to the same cone in $\Sigma(\mathfrak{p})$. Then there exists a facet $\sigma_{0} \in \mathcal{F}(\mathfrak{p})$ such that $F_{\sigma_{0}}(\boldsymbol{a}) \cdot F_{\sigma_{0}}(\boldsymbol{b})<0$. By Theorem $4.5(2), \operatorname{deg}_{\sigma_{0}}(\boldsymbol{a})+\operatorname{deg}_{\sigma_{0}}(\boldsymbol{b})>$ $\operatorname{deg}_{\sigma_{0}}(\boldsymbol{a}+\boldsymbol{b})$.

(2) Let $\boldsymbol{a}, \boldsymbol{b} \in S(\mathfrak{p}) \cap \tau$ for some cone $\tau \in \Sigma(\mathfrak{p})$. Then, for any facet $\sigma \in \mathcal{F}(\mathfrak{p})$, we have $F_{\sigma}(\boldsymbol{a}), F_{\sigma}(\boldsymbol{b}) \in F_{\sigma}(\mathbb{N} A)$ or $F_{\sigma}(\boldsymbol{a}), F_{\sigma}(\boldsymbol{b}) \in-F_{\sigma}(\mathbb{N} A)$. By Theorem 4.5 (2), for any facet $\sigma \in \mathcal{F}(\mathfrak{p})$, we have $\operatorname{deg}_{\sigma}(\boldsymbol{a}+\boldsymbol{b})=\operatorname{deg}_{\sigma}(\boldsymbol{a})+\operatorname{deg}_{\sigma}(\boldsymbol{b})$.

(3) Let $\boldsymbol{a}, \boldsymbol{b} \in \tau \in \Sigma(\mathfrak{p})$. Then $F_{\sigma}(\boldsymbol{a}) \cdot F_{\sigma}(\boldsymbol{b}) \geq 0$ for all $\sigma \in \mathcal{F}(\mathfrak{p})$. Hence, by Theorem 4.5 (2) and (3), for a facet $\sigma \in \mathcal{F}(\mathfrak{p}), \operatorname{deg}_{\sigma}(\boldsymbol{a})+\operatorname{deg}_{\sigma}(\boldsymbol{b})>\operatorname{deg}_{\sigma}(\boldsymbol{a}+\boldsymbol{b})$ if and only if $F_{\sigma}(\boldsymbol{a}), F_{\sigma}(\boldsymbol{b}) \neq 0$, and $\left|F_{\sigma}(\boldsymbol{a})\right|$ or $\left|F_{\sigma}(\boldsymbol{b})\right|$ does not belong to $F_{\sigma}(\mathbb{N} A)$.

In the rest of this section, we fix a prime ideal $\mathfrak{p}$ of $K[s]$. We shall describe all the $\mathbb{Z}^{d}$-graded prime ideals $\mathfrak{P}=\bigoplus_{\boldsymbol{a} \in \mathbb{Z}^{d}} \mathfrak{P}_{\boldsymbol{a}}$ of $G$ with $\mathfrak{P}_{\mathbf{0}}=\mathfrak{p}$.

For a cone $\tau \in \Sigma(\mathfrak{p})$, define a $K$-subspace $\mathfrak{P}(\mathfrak{p}, \tau)=\bigoplus_{\boldsymbol{a} \in \mathbb{Z}^{d}} \mathfrak{P}(\mathfrak{p}, \tau)_{\boldsymbol{a}}$ of $G$ by

$$
\mathfrak{P}(\mathfrak{p}, \tau)_{\boldsymbol{a}}:= \begin{cases}G_{\boldsymbol{a}} \mathfrak{p} & (\boldsymbol{a} \in \tau \cap S(\mathfrak{p})) \\ G_{\boldsymbol{a}} & \text { (otherwise). }\end{cases}
$$

Proposition 4.8. The $K$-subspace $\mathfrak{P}(\mathfrak{p}, \tau)$ is a $\mathbb{Z}^{d}$-graded prime ideal of $G$.

Proof. In order to prove that $\mathfrak{P}(\mathfrak{p}, \tau)$ is an ideal, we need to check

$$
G_{\boldsymbol{a}} \mathfrak{P}(\mathfrak{p}, \tau)_{\boldsymbol{b}} \subseteq \mathfrak{P}(\mathfrak{p}, \tau)_{\boldsymbol{a}+\boldsymbol{b}}
$$

for all $\boldsymbol{a}, \boldsymbol{b} \in \mathbb{Z}^{d}$. If $\boldsymbol{a} \in \tau \cap S(\mathfrak{p})$ or $\boldsymbol{b} \in \tau \cap S(\mathfrak{p})$ or $\boldsymbol{a}+\boldsymbol{b} \notin \tau \cap S(\mathfrak{p})$, then (4.13) is clear from (4.12).

Suppose that $\boldsymbol{a} \notin \tau \cap S(\mathfrak{p}), \boldsymbol{b} \notin \tau \cap S(\mathfrak{p})$, and $\boldsymbol{a}+\boldsymbol{b} \in \tau \cap S(\mathfrak{p})$. Furthermore we may assume that $\boldsymbol{a}$ and $\boldsymbol{b}$ belong to the same cone by Lemma 4.7 (1). Then $\boldsymbol{a}$ and $\boldsymbol{b}$ must belong to $\tau$, since $\boldsymbol{a}+\boldsymbol{b} \in \tau$. Suppose that $\overline{P_{\boldsymbol{a}}} \cdot \overline{P_{\boldsymbol{b}}} \notin G \mathfrak{p}$. Then by Lemma 4.7 (3) there exists a facet $\sigma \in \mathcal{F}(\mathfrak{p})$ such that $F_{\sigma}(\boldsymbol{a})=0$ and $\left|F_{\sigma}(\boldsymbol{b})\right| \notin F_{\sigma}(\mathbb{N} A)$ (or $F_{\sigma}(\boldsymbol{b})=0$ and $\left.\left|F_{\sigma}(\boldsymbol{a})\right| \notin F_{\sigma}(\mathbb{N} A)\right)$, since $\boldsymbol{a} \notin \tau \cap S(\mathfrak{p})$ and $\boldsymbol{b} \notin \tau \cap S(\mathfrak{p})$. Then $\left|F_{\sigma}(\boldsymbol{a}+\boldsymbol{b})\right| \notin F_{\sigma}(\mathbb{N} A)$, which contradicts $\boldsymbol{a}+\boldsymbol{b} \in S(\mathfrak{p})$. Hence $\overline{P_{\boldsymbol{a}}} \cdot \overline{P_{\boldsymbol{b}}} \in G \mathfrak{p}$, and we have proved that $\mathfrak{P}(\mathfrak{p}, \tau)$ is an ideal. 
We have $G / \mathfrak{P}(\mathfrak{p}, \tau)=\bigoplus_{\boldsymbol{a} \in \tau \cap S(\mathfrak{p})} R \overline{P_{\boldsymbol{a}}}$, where $R=K[s] / \mathfrak{p}$ is an integral domain. By Lemma $4.7(2), G / \mathfrak{P}(\mathfrak{p}, \tau)$ is an integral domain. Hence $\mathfrak{P}(\mathfrak{p}, \tau)$ is prime.

Theorem 4.9. Suppose that $\mathfrak{P}=\bigoplus_{\boldsymbol{a} \in \mathbb{Z}^{d}} \mathfrak{P}_{\boldsymbol{a}}$ is a $\mathbb{Z}^{d}$-graded prime ideal of $G$ with $\mathfrak{P}_{\mathbf{0}}=\mathfrak{p}$. Then there exists $\tau \in \Sigma(\mathfrak{p})$ such that $\mathfrak{P}=\mathfrak{P}(\mathfrak{p}, \tau)$. Hence

$$
\pi^{-1}(\mathfrak{p})=\{\mathfrak{P}(\mathfrak{p}, \tau): \tau \in \Sigma(\mathfrak{p})\},
$$

where $\pi$ was defined in (2.8).

Set

$$
S(\mathfrak{P}):=\left\{\boldsymbol{a} \in \mathbb{Z}^{d}: \bar{P}_{\boldsymbol{a}} \notin \mathfrak{P}_{\boldsymbol{a}}\right\} .
$$

We prove three lemmas to prove Theorem 4.9.

Lemma 4.10. Suppose that $\mathfrak{P}=\bigoplus_{\boldsymbol{a} \in \mathbb{Z}^{d}} \mathfrak{P}_{\boldsymbol{a}}$ is a $\mathbb{Z}^{d}$-graded prime ideal of $G$ with $\mathfrak{P}_{\mathbf{0}}=\mathfrak{p}$. Then

$$
S(\mathfrak{P}) \subseteq S(\mathfrak{p})
$$

where $S(\mathfrak{p})$ was defined in (4.8).

Proof. Let $\boldsymbol{a} \notin S(\mathfrak{p})$. Then there exists a facet $\sigma_{0} \in \mathcal{F}(\mathfrak{p})$ such that $\operatorname{deg}_{\sigma_{0}}(\boldsymbol{a})>$ $\operatorname{expdeg}_{\sigma_{0}}(\boldsymbol{a}) \geq 0$ by Corollary 4.4. By Theorem $4.5(2)$,

$$
2 \operatorname{deg}_{\sigma_{0}}(\boldsymbol{a})>\operatorname{deg}_{\sigma_{0}}(2 \boldsymbol{a}) .
$$

Hence

$$
F_{\sigma_{0}} \bar{P}_{2 a} \mid \bar{P}_{a}^{2}
$$

Since $F_{\sigma_{0}} \in \mathfrak{p} \subseteq \mathfrak{P}$, we have $\bar{P}_{\boldsymbol{a}}^{2} \in \mathfrak{P}$. By the primality of $\mathfrak{P}$, we have $\bar{P}_{\boldsymbol{a}} \in \mathfrak{P}$.

Lemma 4.11. Suppose that $\mathfrak{P}=\bigoplus_{\boldsymbol{a} \in \mathbb{Z}^{d}} \mathfrak{P}_{\boldsymbol{a}}$ is a $\mathbb{Z}^{d}$-graded prime ideal of $G$ with $\mathfrak{P}_{\mathbf{0}}=\mathfrak{p}$. Then there exists a cone $\tau \in \Sigma(\mathfrak{p})$ such that $S(\mathfrak{P}) \subseteq \tau \cap S(\mathfrak{p})$.

Proof. Suppose the contrary. Then there exist $\boldsymbol{a}, \boldsymbol{b} \in S(\mathfrak{P})$ and a facet $\sigma_{0} \in \mathcal{F}(\mathfrak{p})$ such that $F_{\sigma_{0}}(\boldsymbol{a})>0, F_{\sigma_{0}}(\boldsymbol{b})<0$. By Theorem $4.5(2)$,

$$
\operatorname{deg}_{\sigma_{0}}(\boldsymbol{a}+\boldsymbol{b})<\operatorname{deg}_{\sigma_{0}}(\boldsymbol{a})+\operatorname{deg}_{\sigma_{0}}(\boldsymbol{b}) \text {. }
$$

Hence

$$
F_{\sigma_{0}} \bar{P}_{\boldsymbol{a}+\boldsymbol{b}} \mid \bar{P}_{\boldsymbol{a}} \bar{P}_{\boldsymbol{b}}
$$

Since $F_{\sigma_{0}} \in \mathfrak{p} \subseteq \mathfrak{P}$, we have $\bar{P}_{\boldsymbol{a}} \bar{P}_{\boldsymbol{b}} \in \mathfrak{P}$. By the primality of $\mathfrak{P}$, we have $\bar{P}_{\boldsymbol{a}}$ or $\bar{P}_{\boldsymbol{b}} \in \mathfrak{P}$, contradicting $\boldsymbol{a}, \boldsymbol{b} \in S(\mathfrak{P})$.

Lemma 4.12. Suppose that $\mathfrak{P}=\bigoplus_{\boldsymbol{a} \in \mathbb{Z}^{d}} \mathfrak{P}_{\boldsymbol{a}}$ is a $\mathbb{Z}^{d}$-graded prime ideal of $G$ with $\mathfrak{P}_{\mathbf{0}}=\mathfrak{p}$. Then

$$
\mathfrak{P}_{\boldsymbol{a}}=G_{\boldsymbol{a}} \mathfrak{p} \quad \text { for all } \boldsymbol{a} \in S(\mathfrak{P}) .
$$

Proof. Let $\boldsymbol{a} \in S(\mathfrak{P})$, and let $\bar{P}_{\boldsymbol{a}} f(s) \in \mathfrak{P}_{\boldsymbol{a}}$. By the primality of $\mathfrak{P}$, we have $f(s) \in \mathfrak{P}$. Hence $f(s) \in \mathfrak{P}_{0}=\mathfrak{p}$.

Now we prove Theorem 4.9 . 
Proof. By Lemma 4.11, there exists a cone $\tau \in \Sigma(\mathfrak{p})$ such that $S(\mathfrak{P}) \subseteq \tau \cap S(\mathfrak{p})$.

For $\epsilon \in\{ \pm 1\}^{\mathcal{F} \backslash \mathcal{F}(\mathfrak{p})}$, put

$$
\tau_{\epsilon}=\left\{\boldsymbol{a} \in \tau: F_{\sigma}(\boldsymbol{a}) \in \mathbb{R}_{\epsilon_{\sigma}} \text { for } \sigma \notin \mathcal{F}(\mathfrak{p})\right\},
$$

where $\mathbb{R}_{+1}=\mathbb{R}_{\geq 0}$ and $\mathbb{R}_{-1}=\mathbb{R}_{\leq 0}$. Then $\tau_{\epsilon} \cap S(\mathfrak{p})$ is finitely generated by the similar argument to that in (Saito, Traves, 2004, §4). Hence $\tau \cap S(\mathfrak{p})=\bigcup_{\epsilon} \tau_{\epsilon} \cap S(\mathfrak{p})$ is also finitely generated, i.e., an affine semigroup. Since $K[\tau \cap S(\mathfrak{p})]:=\bigoplus_{\boldsymbol{a} \in \tau \cap S(\mathfrak{p})} K \bar{P}_{\boldsymbol{a}}$ is a subalgebra of $G, \mathfrak{P} \cap K[\tau \cap S(\mathfrak{p})]$ is a prime of $K[\tau \cap S(\mathfrak{p})]$. Hence by the standard argument (e.g. see (Ishida, 1987, Proposition 1.3)), there exists a face $\nu$ of $\tau$ such that $S(\mathfrak{P})=\nu \cap \tau \cap S(\mathfrak{p})=\nu \cap S(\mathfrak{p})$. Hence $\mathfrak{P}=\mathfrak{P}(\mathfrak{p}, \nu)$.

The following proposition is straightforward.

Proposition 4.13. $\mathfrak{P}(\mathfrak{p}, \tau) \subseteq \mathfrak{P}\left(\mathfrak{p}^{\prime}, \tau^{\prime}\right)$ if and only if $\mathfrak{p} \subseteq \mathfrak{p}^{\prime}$ and $\tau \supseteq \tau^{\prime}$.

The semigroup algebra $R_{A}=K[\mathbb{N} A]$ is a $\mathbb{Z}^{d}$-graded subalgebra of $G$. Hence we have a natural map

$$
\pi_{R_{A}}: \operatorname{Spec}_{\mathbb{Z}^{d}}(G) \ni \mathfrak{P} \mapsto \mathfrak{P} \cap R_{A} \in \operatorname{Spec}_{\mathbb{Z}^{d}}\left(R_{A}\right) .
$$

We know (see e.g. (Ishida, 1987, Proposition 1.3))

$$
\operatorname{Spec}_{\mathbb{Z}^{d}}\left(R_{A}\right)=\left\{\mathfrak{p}_{\tau}: \tau \text { is a face of the cone } \mathbb{R}_{\geq 0} A\right\}
$$

where $\mathfrak{p}_{\tau}=\bigoplus_{a \in \mathbb{N} A \backslash \tau} K t^{a}$.

\section{Proposition 4.14.}

$$
\pi_{R_{A}}^{-1}\left(\mathfrak{p}_{\tau}\right)=\left\{\mathfrak{P}(\mathfrak{q}, \nu): \nu \cap \mathbb{R}_{\geq 0} A=\tau\right\}
$$

Proof. By definition,

$$
\bar{P}_{\boldsymbol{a}} \in \mathfrak{P}(\mathfrak{q}, \nu) \Leftrightarrow \boldsymbol{a} \notin S(\mathfrak{q}) \cap \nu
$$

Hence

$$
\mathfrak{P}(\mathfrak{q}, \nu) \cap R_{A}=\bigoplus_{\boldsymbol{a} \in \mathbb{N} A \backslash S(\mathfrak{q}) \cap \nu} K t^{\boldsymbol{a}}=\bigoplus_{\boldsymbol{a} \in \mathbb{N} A \backslash \nu} K t^{\boldsymbol{a}} .
$$

Here the latter equality holds, since $\boldsymbol{a} \in \mathbb{N} A$ implies $\boldsymbol{a} \in S(\mathfrak{q})$. Therefore,

$$
\mathfrak{P}(\mathfrak{q}, \nu) \cap R_{A}=\mathfrak{p}_{\tau} \Leftrightarrow \mathbb{N} A \backslash \nu=\mathbb{N} A \backslash \tau \Leftrightarrow \mathbb{R}_{\geq 0} A \cap \nu=\tau .
$$

\section{Critical Modules}

Let $R$ be a left Noetherian ring. An $R$-module $M$ of Krull dimension $\delta$ is said to be $\delta$-critical if the Krull dimension of $M / N$ is less than $\delta$ for all nonzero $R$-submodules $N$ of $M$. The 0 -critical modules are precisely the simple modules. Critical modules play a fundamental role in the theory of Noetherian modules. (See for example (Goodearl, Warfield, 1989; Lenagan, 2000; McConnel, Robson, 1987).) In this section, we recall the critical $\mathbb{Z}^{d}$-graded $D\left(R_{A}\right)$-modules studied in (Saito, 2008).

Let $M=\bigoplus_{\boldsymbol{a} \in \mathbb{Z}^{d}} M_{\boldsymbol{a}}$ be a $\mathbb{Z}^{d}$-graded finitely generated left $D\left(R_{A}\right)$-module. Then its Krull dimension $\operatorname{Kdim} M$ in the category of $\mathbb{Z}^{d}$-graded finitely generated left $D\left(R_{A}\right)$ modules is given by $\operatorname{Kdim} M=\max _{\boldsymbol{a}} \operatorname{dim}_{K[s]} M_{\boldsymbol{a}}$ (Saito, 2008, Theorem 6.1).

For an ideal $I$ of $K[s]$ and $\boldsymbol{\beta} \in K^{d}$, we define a new ideal $I+\boldsymbol{\beta}$ by

$$
I+\boldsymbol{\beta}:=\{f(s-\boldsymbol{\beta}): f(s) \in I\} .
$$


Let $\mathfrak{p}$ be a prime ideal of $K[s]$.

For $\boldsymbol{c} \in \mathbb{Z}^{d}$, we write $\mathfrak{p} \preceq \mathfrak{p}+\boldsymbol{c}$ if $\mathbb{I}(\Omega(\boldsymbol{c})) \nsubseteq \mathbb{p}$, and $\mathfrak{p} \sim \mathfrak{p}+\boldsymbol{c}$ if $\mathfrak{p} \preceq \mathfrak{p}+\boldsymbol{c}$ and $\mathfrak{p}+\boldsymbol{c} \preceq \mathfrak{p}$. Then $\sim$ is an equivalence relation in

$$
[\mathfrak{p}]:=\left\{\mathfrak{p}+\boldsymbol{c}: \boldsymbol{c} \in \mathbb{Z}^{d}\right\}
$$

and $[\mathfrak{p}] / \sim$ is finite (Saito, 2008, Corollary 3.6).

Let $\delta=\operatorname{dim} K[s] / \mathfrak{p}$. Let $L(\mathfrak{p})=\bigoplus_{\boldsymbol{a} \in \mathbb{Z}^{d}} L(\mathfrak{p})_{\boldsymbol{a}}$ be the cyclic $\mathbb{Z}^{d}$-graded left $D\left(R_{A}\right)$ module $L(\mathfrak{p})=D\left(R_{A}\right) / I(\mathfrak{p})$ with

$$
I(\mathfrak{p})_{\boldsymbol{a}}= \begin{cases}t^{\boldsymbol{a}}(\mathbb{I}(\Omega(\boldsymbol{a})) \cap \mathfrak{p}) & (\mathfrak{p} \sim \mathfrak{p}+\boldsymbol{a}) \\ D\left(R_{A}\right)_{\boldsymbol{a}} & \text { (otherwise) }\end{cases}
$$

Then $L(\mathfrak{p})$ is $\delta$-critical (Saito, 2008, Example 7.5).

Conversely, let $M$ be a $\delta$-critical $\mathbb{Z}^{d}$-graded left $D\left(R_{A}\right)$-module generated by one element $v \in M_{\mathbf{0}}$ with $\operatorname{Ann}_{K[s]}(v)=\mathfrak{p}$. Then $M$ is isomorphic to $L(\mathfrak{p})$ (Saito, 2008, Theorem 7.7).

\section{Characteristic Varieties of Critical Modules}

For a cyclic $D$-module $D / I$, the support of the $\operatorname{Gr} D$-module $\operatorname{Gr} D / \operatorname{Gr} I$, where $\operatorname{Gr} I=$ $\bigoplus_{k=0}^{\infty} I \cap D_{k} / I \cap D_{k-1}$, is called the characteristic variety of $D / I$. For details about characteristic varieties, see any textbook of the theory of $D$-modules, for example, (Björk, 1979; Borel et al, 1987; Coutinho, 1995; Hotta, Takeuchi, Tanisaki, 2008; Kashiwara, 2003).

In this section, we fix a prime ideal $\mathfrak{p}$ of $K[s]$ homogeneous with respect to $s_{1}, \ldots, s_{d}$, and a vector $\boldsymbol{\beta} \in K^{d}$, and we consider the characteristic variety of the critical $\mathbb{Z}^{d}$-graded $D\left(R_{A}\right)$-module $L(\mathfrak{p}+\boldsymbol{\beta})$ (for the definition, see (5.1) and (5.2)). Note that

$$
\mathfrak{p} \subseteq \mathfrak{m}:=\left(s_{1}, \ldots, s_{d}\right) .
$$

By definition,

$$
I(\mathfrak{p}+\boldsymbol{\beta})_{\boldsymbol{a}}= \begin{cases}t^{\boldsymbol{a}}(\mathbb{I}(\Omega(\boldsymbol{a})) \cap(\mathfrak{p}+\boldsymbol{\beta})) & (\mathfrak{p}+\boldsymbol{\beta} \sim \mathfrak{p}+\boldsymbol{\beta}+\boldsymbol{a}) \\ D\left(R_{A}\right)_{\boldsymbol{a}} & (\mathfrak{p}+\boldsymbol{\beta} \neq \mathfrak{p}+\boldsymbol{\beta}+\boldsymbol{a}) .\end{cases}
$$

If $\mathfrak{p}+\boldsymbol{\beta} \sim \mathfrak{p}+\boldsymbol{\beta}+\boldsymbol{a}$, then $\mathbb{I}(\Omega(\boldsymbol{a})) \nsubseteq \mathfrak{p}+\boldsymbol{\beta}$. Recall that $\mathbb{I}(\Omega(\boldsymbol{a}))$ is generated by $p_{\boldsymbol{a}}$ (Corollary $2.4)$. Hence, if $\mathfrak{p}+\boldsymbol{\beta} \sim \mathfrak{p}+\boldsymbol{\beta}+\boldsymbol{a}$, then $p_{\boldsymbol{a}} \notin \mathfrak{p}+\boldsymbol{\beta}$, and $\mathbb{I}(\Omega(\boldsymbol{a})) \cap(\mathfrak{p}+\boldsymbol{\beta})=\mathbb{I}(\Omega(\boldsymbol{a})) \cdot(\mathfrak{p}+\boldsymbol{\beta})$. Thus

$$
I(\mathfrak{p}+\boldsymbol{\beta})_{\boldsymbol{a}}= \begin{cases}D\left(R_{A}\right)_{\boldsymbol{a}} \cdot(\mathfrak{p}+\boldsymbol{\beta}) & (\mathfrak{p}+\boldsymbol{\beta} \sim \mathfrak{p}+\boldsymbol{\beta}+\boldsymbol{a}) \\ D\left(R_{A}\right)_{\boldsymbol{a}} & (\mathfrak{p}+\boldsymbol{\beta} \sim \mathfrak{p}+\boldsymbol{\beta}+\boldsymbol{a}) .\end{cases}
$$

We have $\operatorname{Gr} L(\mathfrak{p}+\boldsymbol{\beta})=\operatorname{Gr} D\left(R_{A}\right) / \operatorname{Gr} I(\mathfrak{p}+\boldsymbol{\beta})=G / \operatorname{Gr} I(\mathfrak{p}+\boldsymbol{\beta})$, and

$$
\operatorname{Gr} I(\mathfrak{p}+\boldsymbol{\beta})_{\boldsymbol{a}}= \begin{cases}G_{\boldsymbol{a}} \cdot \mathfrak{p} & (\mathfrak{p}+\boldsymbol{\beta} \sim \mathfrak{p}+\boldsymbol{\beta}+\boldsymbol{a}) \\ G_{\boldsymbol{a}} & (\mathfrak{p}+\boldsymbol{\beta} \nsim \mathfrak{p}+\boldsymbol{\beta}+\boldsymbol{a}) .\end{cases}
$$

Lemma 6.1. The equivalence class of $\mathfrak{p}+\boldsymbol{\beta}+\boldsymbol{a}$ with respect to $\sim$ is determined by the sets $\left\{\sigma \in \mathcal{F}(\mathfrak{p}): F_{\sigma}(\boldsymbol{\beta}+\boldsymbol{a}) \in F_{\sigma}(\mathbb{N} A)\right\}$ and $\left\{\sigma \in \mathcal{F}(\mathfrak{p}): F_{\sigma}(\boldsymbol{\beta}+\boldsymbol{a}) \in \mathbb{Z} \backslash F_{\sigma}(\mathbb{N} A)\right\}$, namely, for $\boldsymbol{a}, \boldsymbol{b} \in \mathbb{Z}^{d}, \mathfrak{p}+\boldsymbol{\beta}+\boldsymbol{a} \sim \mathfrak{p}+\boldsymbol{\beta}+\boldsymbol{b}$ if and only if

$$
\left\{\sigma \in \mathcal{F}(\mathfrak{p}): F_{\sigma}(\boldsymbol{\beta}+\boldsymbol{a}) \in F_{\sigma}(\mathbb{N} A)\right\}=\left\{\sigma \in \mathcal{F}(\mathfrak{p}): F_{\sigma}(\boldsymbol{\beta}+\boldsymbol{b}) \in F_{\sigma}(\mathbb{N} A)\right\}
$$

and

$$
\left\{\sigma \in \mathcal{F}(\mathfrak{p}): F_{\sigma}(\boldsymbol{\beta}+\boldsymbol{a}) \in \mathbb{Z} \backslash F_{\sigma}(\mathbb{N} A)\right\}=\left\{\sigma \in \mathcal{F}(\mathfrak{p}): F_{\sigma}(\boldsymbol{\beta}+\boldsymbol{b}) \in \mathbb{Z} \backslash F_{\sigma}(\mathbb{N} A)\right\} .
$$


Proof. Put

$$
\mathcal{F}(\mathfrak{p}, \boldsymbol{\beta})_{\mathbb{Z}}:=\left\{\sigma \in \mathcal{F}(\mathfrak{p}): F_{\sigma}(\boldsymbol{\beta}) \in \mathbb{Z}\right\}
$$

By definition, $\mathfrak{p}+\boldsymbol{\beta}+\boldsymbol{a} \sim \mathfrak{p}+\boldsymbol{\beta}+\boldsymbol{b}$ means $p_{\boldsymbol{b}-\boldsymbol{a}} \notin \mathfrak{p}+\boldsymbol{\beta}+\boldsymbol{a}$ and $p_{\boldsymbol{a}-\boldsymbol{b}} \notin \mathfrak{p}+\boldsymbol{\beta}+\boldsymbol{b}$, since $\mathbb{I}(\Omega(\boldsymbol{b}-\boldsymbol{a}))$ and $\mathbb{I}(\Omega(\boldsymbol{a}-\boldsymbol{b}))$ are generated by $p_{\boldsymbol{b}-\boldsymbol{a}}$ and $p_{\boldsymbol{a}-\boldsymbol{b}}$, respectively (Corollary 2.4).

First note that $F_{\sigma}-l \notin \mathfrak{p}+\boldsymbol{\beta}+\boldsymbol{a}$ for any $l \in \mathbb{Z}$ if $F_{\sigma}(\boldsymbol{\beta}) \notin \mathbb{Z}$, since in this case $F_{\sigma}(\boldsymbol{\beta}+\boldsymbol{a}) \neq l$, or equivalently $F_{\sigma}-l \notin \mathfrak{m}+\boldsymbol{\beta}+\boldsymbol{a}$ (see (6.1) for $\mathfrak{m}$ ). Next note that $F_{\sigma}-l \notin \mathfrak{p}+\boldsymbol{\beta}+\boldsymbol{a}$ for any $\sigma \notin \mathcal{F}(\mathfrak{p})$ and any $l \in \mathbb{Z}$, since otherwise we have $F_{\sigma} \in \mathfrak{p}$ by taking Gr.

Hence $p_{\boldsymbol{b}-\boldsymbol{a}} \notin \mathfrak{p}+\boldsymbol{\beta}+\boldsymbol{a}$ if and only if

$$
\prod_{\sigma \in \mathcal{F}(\mathfrak{p}, \boldsymbol{\beta})_{\mathbb{Z}}} \prod_{k \in F_{\sigma}(\mathbb{N} A) \backslash\left(F_{\sigma}(\boldsymbol{a})-F_{\sigma}(\boldsymbol{b})+F_{\sigma}(\mathbb{N} A)\right)}\left(F_{\sigma}-k\right) \notin \mathfrak{p}+\boldsymbol{\beta}+\boldsymbol{a} .
$$

This is equivalent to the condition: If $\sigma \in \mathcal{F}(\mathfrak{p}, \boldsymbol{\beta})_{\mathbb{Z}}, k \in F_{\sigma}(\mathbb{N} A)$, and $k \notin F_{\sigma}(\boldsymbol{a})-$ $F_{\sigma}(\boldsymbol{b})+F_{\sigma}(\mathbb{N} A)$, then $F_{\sigma}(s+\boldsymbol{\beta}+\boldsymbol{a})-k \notin \mathfrak{p}$. Since $F_{\sigma}(s+\boldsymbol{\beta}+\boldsymbol{a})-k \notin \mathfrak{p}$ is equivalent to $F_{\sigma}(\boldsymbol{\beta}+\boldsymbol{a}) \neq k$, it follows that $p_{\boldsymbol{b}-\boldsymbol{a}} \notin \mathfrak{p}+\boldsymbol{\beta}+\boldsymbol{a}$ is equivalent to the condition: For each $\sigma \in \mathcal{F}(\mathfrak{p}, \boldsymbol{\beta})_{\mathbb{Z}}, F_{\sigma}(\boldsymbol{\beta}+\boldsymbol{a}) \in \mathbb{Z} \backslash F_{\sigma}(\mathbb{N} A)$ or $F_{\sigma}(\boldsymbol{\beta}+\boldsymbol{a}) \in F_{\sigma}(\boldsymbol{a})-F_{\sigma}(\boldsymbol{b})+F_{\sigma}(\mathbb{N} A)$ (or $\left.F_{\sigma}(\boldsymbol{\beta}+\boldsymbol{b}) \in F_{\sigma}(\mathbb{N} A)\right)$.

Therefore $\mathfrak{p}+\boldsymbol{\beta}+\boldsymbol{a} \sim \mathfrak{p}+\boldsymbol{\beta}+\boldsymbol{b}$ if and only if for each $\sigma \in \mathcal{F}(\mathfrak{p}, \boldsymbol{\beta})_{\mathbb{Z}}\left(F_{\sigma}(\boldsymbol{\beta}+\boldsymbol{a}) \in\right.$ $\mathbb{Z} \backslash F_{\sigma}(\mathbb{N} A)$ or $\left.F_{\sigma}(\boldsymbol{\beta}+\boldsymbol{b}) \in F_{\sigma}(\mathbb{N} A)\right)$ and $\left(F_{\sigma}(\boldsymbol{\beta}+\boldsymbol{b}) \in \mathbb{Z} \backslash F_{\sigma}(\mathbb{N} A)\right.$ or $\left.F_{\sigma}(\boldsymbol{\beta}+\boldsymbol{a}) \in F_{\sigma}(\mathbb{N} A)\right)$.

Hence $\mathfrak{p}+\boldsymbol{\beta}+\boldsymbol{a} \sim \mathfrak{p}+\boldsymbol{\beta}+\boldsymbol{b}$ if and only if for each $\sigma \in \mathcal{F}(\mathfrak{p}, \boldsymbol{\beta})_{\mathbb{Z}}\left(F_{\sigma}(\boldsymbol{\beta}+\boldsymbol{a}) \in \mathbb{Z} \backslash F_{\sigma}(\mathbb{N} A)\right.$ and $\left.F_{\sigma}(\boldsymbol{\beta}+\boldsymbol{b}) \in \mathbb{Z} \backslash F_{\sigma}(\mathbb{N} A)\right)$ or $\left(F_{\sigma}(\boldsymbol{\beta}+\boldsymbol{a}) \in F_{\sigma}(\mathbb{N} A)\right.$ and $\left.F_{\sigma}(\boldsymbol{\beta}+\boldsymbol{b}) \in F_{\sigma}(\mathbb{N} A)\right)$.

Let $\tau(\mathfrak{p}+\boldsymbol{\beta})$ denote the cone defined by

$$
\tau(\mathfrak{p}+\boldsymbol{\beta}):=\bigcap_{\sigma \in \mathcal{F}(\mathfrak{p}, \boldsymbol{\beta})_{\mathbb{Z}} ; F_{\sigma}(\boldsymbol{\beta}) \in F_{\sigma}(\mathbb{N} A)}\left(F_{\sigma} \geq 0\right) \cap \bigcap_{\sigma \in \mathcal{F}(\mathfrak{p}, \boldsymbol{\beta})_{\mathbb{Z}} ; F_{\sigma}(\boldsymbol{\beta}) \notin F_{\sigma}(\mathbb{N} A)}\left(F_{\sigma} \leq 0\right),
$$

where $\mathcal{F}(\mathfrak{p}, \boldsymbol{\beta})_{\mathbb{Z}}$ was defined in (6.3). Then $\tau(\mathfrak{p}+\boldsymbol{\beta})$ is a union of some cones in $\Sigma(\mathfrak{p})$ by definition (4.7).

Theorem 6.2.

(1)

$$
\sqrt{\operatorname{Gr} I(\mathfrak{p}+\boldsymbol{\beta})}=\bigcap_{\tau \in \Sigma(\mathfrak{p}), \tau \subseteq \tau(\mathfrak{p}+\boldsymbol{\beta})} \mathfrak{P}(\mathfrak{p}, \tau)
$$

namely

$$
(\sqrt{\operatorname{Gr} I(\mathfrak{p}+\boldsymbol{\beta})})_{\boldsymbol{a}}= \begin{cases}G_{\boldsymbol{a}} \cdot \mathfrak{p} & (\boldsymbol{a} \in \tau(\mathfrak{p}+\boldsymbol{\beta}) \cap S(\mathfrak{p})) \\ G_{\boldsymbol{a}} & (\text { otherwise }) .\end{cases}
$$

(2) The characteristic variety of $L(\mathfrak{p}+\boldsymbol{\beta})=D\left(R_{A}\right) / I(\mathfrak{p}+\boldsymbol{\beta})$ is irreducible if and only if $\tau(\mathfrak{p}+\boldsymbol{\beta}) \in \Sigma(\mathfrak{p})$. In this case,

$$
\sqrt{\operatorname{Gr} I(\mathfrak{p}+\boldsymbol{\beta})}=\mathfrak{P}(\mathfrak{p}, \tau(\mathfrak{p}+\boldsymbol{\beta})) .
$$

(3) If $F_{\sigma}(\boldsymbol{\beta}) \in \mathbb{Z}$ for all $\sigma \in \mathcal{F}(\mathfrak{p})$, then the characteristic variety of $L(\mathfrak{p}+\boldsymbol{\beta})$ is irreducible.

Proof. (1) By the definition of $\mathfrak{P}(\mathfrak{p}, \tau)$ (4.12), (6.5) immediately follows from (6.6). We prove (6.6). Let $\boldsymbol{a} \notin \tau(\mathfrak{p}+\boldsymbol{\beta})$ and $\boldsymbol{a} \in S(\mathfrak{p})$ (see (4.8) for the definition of $S(\mathfrak{p})$ ). Then 
by Lemma 6.1 there exists $m \in \mathbb{N}$ such that $\mathfrak{p}+\boldsymbol{\beta}+m \boldsymbol{a} \not \mathfrak{p}+\boldsymbol{\beta}$. Hence $P_{m \boldsymbol{a}} \in I(\mathfrak{p}+\boldsymbol{\beta})$ by definition. Since $P_{\boldsymbol{a}}^{m}=P_{m \boldsymbol{a}} \cdot f(s)$ for some $f(s) \in K[s]$, we have $\overline{P_{\boldsymbol{a}}} \in \sqrt{\operatorname{Gr} I(\mathfrak{p}+\boldsymbol{\beta})}$.

Next let $\boldsymbol{a} \notin S(\mathfrak{p})$. Then, by Theorem $4.5(2), P_{\boldsymbol{a}}^{2}=P_{2 \boldsymbol{a}} \cdot g(s)$ with $\left(F_{\sigma}-k\right) \mid g(s)$ for some $\sigma \in \mathcal{F}(\mathfrak{p})$ and $k \in \mathbb{Z}$. Hence ${\overline{P_{\boldsymbol{a}}}}^{2}=\overline{P_{2 \boldsymbol{a}}} \cdot \overline{g(s)}$, and $F_{\sigma} \mid \overline{g(s)}$ for some $\sigma \in \mathcal{F}(\mathfrak{p})$. Hence $\overline{g(s)} \in \mathfrak{p}$, and ${\overline{P_{\boldsymbol{a}}}}^{2} \in \operatorname{Gr} I(\mathfrak{p}+\boldsymbol{\beta})$ by (6.2). Therefore we have proved that $\sqrt{\operatorname{Gr} I(\mathfrak{p}+\boldsymbol{\beta})_{\boldsymbol{a}}}=$ $G_{\boldsymbol{a}}$ for $\boldsymbol{a} \notin \tau(\mathfrak{p}+\boldsymbol{\beta}) \cap S(\mathfrak{p})$.

Finally let $\boldsymbol{a} \in \tau(\mathfrak{p}+\boldsymbol{\beta}) \cap S(\mathfrak{p})$. We claim that $\mathfrak{p}+\boldsymbol{\beta}+m \boldsymbol{a} \sim \mathfrak{p}+\boldsymbol{\beta}$ for all $m \in \mathbb{N}$.

Let $\sigma \in \mathcal{F}(\mathfrak{p}, \boldsymbol{\beta})_{\mathbb{Z}}$. Since $\boldsymbol{a} \in \tau(\mathfrak{p}+\boldsymbol{\beta}) \cap S(\mathfrak{p}), F_{\sigma}(\boldsymbol{\beta}) \notin F_{\sigma}(\mathbb{N} A)$ implies $F_{\sigma}(\boldsymbol{a}) \in-F_{\sigma}(\mathbb{N} A)$ and hence $F_{\sigma}(m \boldsymbol{a}) \in-F_{\sigma}(\mathbb{N} A)$. Hence in this case we have $F_{\sigma}(\boldsymbol{\beta}+m \boldsymbol{a}) \notin F_{\sigma}(\mathbb{N} A)$. Similarly $F_{\sigma}(\boldsymbol{\beta}) \in F_{\sigma}(\mathbb{N} A)$ implies $F_{\sigma}(\boldsymbol{a}) \in F_{\sigma}(\mathbb{N} A)$ and hence $F_{\sigma}(\boldsymbol{\beta}+m \boldsymbol{a}) \in F_{\sigma}(\mathbb{N} A)$. Thus by Lemma $6.1 \mathfrak{p}+\boldsymbol{\beta}+m \boldsymbol{a} \sim \mathfrak{p}+\boldsymbol{\beta}$.

Since $\mathfrak{p}+\boldsymbol{\beta}+\boldsymbol{a} \sim \mathfrak{p}+\boldsymbol{\beta}$, we have, by (6.2), Gr $I(\mathfrak{p}+\boldsymbol{\beta})_{\boldsymbol{a}}=G_{\boldsymbol{a}} \cdot \mathfrak{p}$ for $\boldsymbol{a} \in \tau(\mathfrak{p}+\boldsymbol{\beta}) \cap S(\mathfrak{p})$.

Suppose that $\overline{P_{\boldsymbol{a}}} f(s) \in \sqrt{\operatorname{Gr} I(\mathfrak{p}+\boldsymbol{\beta})}$ for a homogeneous polynomial $f(s)$. Then there exists $m \in \mathbb{N}$ such that ${\overline{P_{\boldsymbol{a}}}}^{m} f(s)^{m} \in \mathrm{Gr} I(\mathfrak{p}+\boldsymbol{\beta})_{m \boldsymbol{a}}$. Hence by (6.2) there exists a polynomial $g(s) \in \mathfrak{p}$ such that $\overline{P_{\boldsymbol{a}}} f(s)^{m}=\overline{P_{m \boldsymbol{a}}} g(s)$. Since $\boldsymbol{a} \in S(\mathfrak{p})$, by Theorem 4.5 $(2),{\overline{P_{\boldsymbol{a}}}}^{m}={\overline{P_{m \boldsymbol{a}}}}_{h}(s)$ for some $h(s) \notin \mathfrak{p}$. Hence $h(s) f(s)^{m}=g(s) \in \mathfrak{p}$. Since $\mathfrak{p}$ is prime, $f(s) \in \mathfrak{p}$. This proves $\sqrt{\operatorname{Gr} I(\mathfrak{p}+\boldsymbol{\beta})_{\boldsymbol{a}}} \subseteq G_{\boldsymbol{a}} \cdot \mathfrak{p}$ for $\boldsymbol{a} \in \tau(\mathfrak{p}+\boldsymbol{\beta}) \cap S(\mathfrak{p})$, and completes the proof of $(1)$.

(2) immediately follows from (1).

If $F_{\sigma}(\boldsymbol{\beta}) \in \mathbb{Z}$ for all $\sigma \in \mathcal{F}(\mathfrak{p})$, then $\tau(\mathfrak{p}+\boldsymbol{\beta}) \in \Sigma(\mathfrak{p})$ by definition. Hence (3) follows from $(2)$.

Example 6.3. Let $n=d=1$, and $A=(1)$. In this case, $D\left(R_{A}\right)$ is the first Weyl algebra $K\langle t, \partial\rangle$, and $G=\operatorname{Gr} D\left(R_{A}\right)$ is the polynomial algebra $K[t, \xi]$ :

$$
D\left(R_{A}\right)_{a}=P_{a} K[s], \quad P_{a}= \begin{cases}t^{a} & (a \geq 0) \\ \partial^{-a} & (a<0)\end{cases}
$$

where $s=t \partial$. Note that $\partial^{a}=t^{-a} p_{-a}$, where $p_{-a}=s(s-1) \cdots(s-a+1)$ for $a \in \mathbb{N}$.

The cone $\mathbb{R}_{\geq 0} A=\mathbb{R}_{\geq 0}$ has only one facet $\{0\}$, and $F_{\{0\}}(s)=s$. Let $\mathfrak{m}$ be the maximal ideal $(s)$ of $K[s]$. Then $\Sigma(\mathfrak{m})$ is the fan consisting of $\{0\}, \mathbb{R}_{\geq 0}$, and $\mathbb{R}_{\leq 0}$. Then

$$
\begin{aligned}
\mathfrak{P}(\mathfrak{m},\{0\}) & =(t, \xi, s)=(t, \xi), \\
\mathfrak{P}\left(\mathfrak{m}, \mathbb{R}_{\geq 0}\right) & =(\xi, s)=(\xi), \\
\mathfrak{P}\left(\mathfrak{m}, \mathbb{R}_{\leq 0}\right) & =(t, s)=(t),
\end{aligned}
$$

where $s=t \xi$ in $G$.

Let $\beta \notin \mathbb{Z}$, and consider $I(\mathfrak{m}+\beta)=D\left(R_{A}\right)(s-\beta)$. Since $\tau(\mathfrak{m}+\beta)=\mathbb{R}$, we have $\operatorname{Gr} I(\mathfrak{m}+\beta)=(s)=\mathfrak{P}\left(\mathfrak{m}, \mathbb{R}_{\geq 0}\right) \cap \mathfrak{P}\left(\mathfrak{m}, \mathbb{R}_{\leq 0}\right)$ by Theorem 6.2. Thus the characteristic variety of $L(\mathfrak{m}+\beta)$ has two irreducible components.

Example 6.4. Let $A=\left(\begin{array}{cccccc}1 & 1 & 1 & 1 & 1 & 1 \\ 0 & 3 & 4 & 0 & 3 & 4 \\ 0 & 0 & 0 & 1 & 1 & 1\end{array}\right)=\left(\boldsymbol{a}_{1}, \ldots, \boldsymbol{a}_{6}\right)$. There are four facets: $\sigma_{14}$, $\sigma_{36}, \sigma_{123}, \sigma_{456}$, where $\sigma_{14}=\mathbb{R}_{\geq 0} \boldsymbol{a}_{1}+\mathbb{R}_{\geq 0} \boldsymbol{a}_{4}$, and so on. The primitive integral support functions are $F_{\sigma_{14}}(s)=s_{2}, F_{\sigma_{36}}(s)=4 s_{1}-s_{2}, F_{\sigma_{123}}(s)=s_{3}, F_{\sigma_{456}}(s)=s_{1}-s_{3}$. We have

$$
F_{\sigma_{14}}(\mathbb{N} A)=3 \mathbb{N}+4 \mathbb{N}=\mathbb{N} \backslash\{1,2,5\}, \quad F_{\sigma_{36}}(\mathbb{N} A)=F_{\sigma_{123}}(\mathbb{N} A)=F_{\sigma_{456}}(\mathbb{N} A)=\mathbb{N}
$$


We have the relation

$$
F_{\sigma_{14}}+F_{\sigma_{36}}=4 s_{1}=4\left(F_{\sigma_{123}}+F_{\sigma_{456}}\right) .
$$

The set

$$
\begin{aligned}
& \mathbb{Z}^{3} \cap F_{\sigma_{14}}^{-1}(\mathbb{Z} \backslash[3 \mathbb{N}+4 \mathbb{N}]) \cap F_{\sigma_{36}}^{-1}(\mathbb{Z} \backslash \mathbb{N}) \cap F_{\sigma_{123}}^{-1}(\mathbb{N}) \cap F_{\sigma_{456}}^{-1}(\mathbb{N}) \\
= & \left\{\left(\begin{array}{l}
0 \\
1 \\
0
\end{array}\right),\left(\begin{array}{l}
0 \\
2 \\
0
\end{array}\right),\left(\begin{array}{l}
0 \\
5 \\
0
\end{array}\right),\left(\begin{array}{l}
1 \\
5 \\
0
\end{array}\right),\left(\begin{array}{l}
1 \\
5 \\
1
\end{array}\right)\right\}
\end{aligned}
$$

is an equivalence class (with respect to $\mathfrak{m}=\left(s_{1}, s_{2}, s_{3}\right)$ ) by Lemma 6.1.

Since $\tau\left(\mathfrak{m}+{ }^{t}(0,1,0)\right)=\{0\}$,

$$
\sqrt{\operatorname{Gr} I\left(\mathfrak{m}+{ }^{t}(0,1,0)\right)}=\mathfrak{P}(\mathfrak{m},\{\mathbf{0}\})
$$

by Theorem 6.2. In this case, $\operatorname{Gr} I\left(\mathfrak{m}+{ }^{t}(0,1,0)\right)$ is not radical.

For $\boldsymbol{\beta}=\mathbf{0}$, we do not need to take the radical.

\section{Proposition 6.5.}

$$
\operatorname{Gr} I(\mathfrak{p})=\mathfrak{P}(\mathfrak{p}, \tau(\mathfrak{p})) .
$$

Proof. By Lemma 6.1, $\mathfrak{p} \sim \mathfrak{p}+\boldsymbol{a}$ if and only if $F_{\sigma}(\boldsymbol{a}) \in F_{\sigma}(\mathbb{N} A)$ for all $\sigma \in \mathcal{F}(\mathfrak{p})$, which means $\boldsymbol{a} \in \tau(\mathfrak{p}) \cap S(\mathfrak{p})$. Hence the proposition follows from (4.12) and (6.2).

\section{REFERENCES}

[Björk, J.-E. (1979)] Rings of differential operators. North-Holland, Amsterdam.

[Borel, A. et al. (1987)] Algebraic D-Modules. Academic Press, New York.

[Coutinho, S. C. (1995)] A primer of algebraic D-modules. London Math. Soc. Student Texts 33, Cambridge Univ. Press, Cambridge.

[Fulton, W. (1993)] Introduction to toric varieties. Annals of Math. Studies 131, Princeton Univ. Press, Princeton.

[Goodearl, K.R., Warfield, R.B. (1989)] An introduction to noncommutative Noetherian rings. London Math. Soc. Student Texts 16, Cambridge Univ. Press, Cambridge.

[Hotta, R., Takeuchi, K., Tanisaki, T. (2008)] D-modules, perverse sheaves, and representation theory. Birkhäuser, Basel.

[Ishida, M.-N. (1987)] The local cohomology groups of an affine semigroup ring. In Algebraic Geometry and Commutative Algebra in Honor of Masayoshi NAGATA, vol. 1, Kinokuniya, Tokyo, 141-153.

[Kashiwara, M. (2003)] D-modules and Microlocal Calculus. American Mathematical Society, Providence.

[Lenagan, T. H. (2000)] Dimension theory of Noetherian rings. In: H. Krause, C. Ringel, ed. Infinite length modules. Trends in Math., Birkhäuser, Basel, pp. 129-148.

[McConnel, J. C., Robson, J. C. (1987)] Noncommutative Noetherian rings. John Wiley \& Sons, Chichester.

[Musson, I. M. (1987)] Rings of differential operators on invariants of tori. Trans. Amer. Math. Soc. 303:805-827.

[Musson, I. M., Van den Bergh, M. (1998)] Invariants under tori of rings of differential operators and related topics. Mem. Amer. Math. Soc., 650.

[Saito, M. (2007)] Primitive ideals of the ring of differential operators on an affine toric variety. Tohoku Math. J. 59:119-144.

[Saito, M. (2008)] Critical modules of the ring of differential operators of affine semigroup algebras. Preprint.

[Saito, M., Traves, W. N. (2001)] Differential algebras on semigroup algebras. AMS Contemporary Math. 286:207-226.

[Saito, M., Traves, W. N. (2004)] Finite generations of rings of differential operators of semigroup algebras. J. of Algebra 278:76-103. 
Department of Mathematics

HOKKAIDO UNIVERSITY

SAPPORO, 060-0810 JAPAN

E-mail address: saito@math.sci.hokudai.ac.jp 